\title{
Temporal dynamics of mental impasses underlying insight-like problem solving
}

\author{
SHEN WangBing ${ }^{1}$, LIU Chang ${ }^{1 *}$, YUAN Yuan ${ }^{1}$, ZHANG XiaoJiang ${ }^{1} \&$ LUO Jin $^{2,3 *}$ \\ ${ }^{1}$ Lab of Cognitive Neuroscience and School of Psychology, Nanjing Normal University, Nanjing 210097, China; \\ ${ }^{2}$ Beijing Key Laboratory of Learning and Cognition, Capital Normal University, Beijing 100083, China; \\ ${ }^{3}$ Key Laboratory of Mental Health, Institute of Psychology, Chinese Academy of Sciences, Beijing 100101, China
}

Received November 14, 2012; accepted December 25, 2012

\begin{abstract}
Insight problem solving is characterized by mental impasses, states of mind in which the problem solver does not know what to do next. Although many studies have investigated the neural correlates of insight problem solving, however, the question when mental impasses occur during insight problem solving has been rarely studied. The present study adopted high temporal resolution ERPs to investigate the temporal dynamics of an impasse underlying insight problem solving. Time locked ERPs were recorded associated with problems with impasses (PWI) and problems without impasses (POI). The problem types were determined by participants' subjective responses. The results revealed an early frontocentral P2 was linked with the preconscious awareness of mental impasses and a P3a was associated with fixed attention when the impasse formed. These findings suggest the impasse may occur initially at a relatively early stage and metacognition plays an important role in insight problem solving.
\end{abstract}

insight problem solving, metacognition, mental impasse, event-related potential (ERP), P200

Citation: Shen W B, Liu C, Yuan Y, et al. Temporal dynamics of mental impasses underlying insight-like problem solving. Sci China Life Sci, 2013, 56: 284-290, doi: 10.1007/s11427-013-4454-8

Insight is a topic of growing interest in psychology [1-4]. Insight in problem solving occurs when the problem solver fails to see how to solve a problem and then "Aha" there is a sudden realization of how to solve it. The realization of how to solve the problem is usually preceded by a mental impasse [5], where the solver becomes stuck and cannot see how to solve the problem [6]. The impasse, a key and elementary process within insight, has been rarely investigated.

Mental impasse mainly resulted from the failure of the 'repeated' explorations and occurred at a late stage [7]. In that case, the impasse was defined as the state of mind in which the problem solver felt 'all' options have been explored and he could not think out what to do next [8]. However, increasing studies on insight using high temporal resolution ERPs showed that people overcame the impasse

*Corresponding author (email: cglew@163.com; luoj@psych.ac.cn) at an early stage, rather than at a later stage. Recently, Zhao et al. have found a positivity (P500-700) involving the breaking of mental set after onset of test stimuli [9]. Additionally, many studies also revealed insight problems or solutions as opposed to non-insight problems or solutions elicited an early ERP component (mainly from 200 to 800 $\mathrm{ms}$ ) involving set shifts $[1,10,11]$. Previous studies have indicated it usually lasted about some hundred or thousand milliseconds from the occurrence of an impasse to set shifts [12]. Hence, they suggested the mental impasse may occur at an early, even at the perceptual stage.

Additionally, some theories on insight also implied mental impasses might appear at an early stage. For example, the functional fixedness hypothesis claims the impasse results from the 'spontaneous' retrieval of familiar usage in a problem that need to be solved by a familiar object in an unfamiliar way [12-14]. Representation change theory 
thinks people encounter impasses because their initial representations are misleading [13] or incomplete [5]. According to traditional Gestalt theory, thinking begins when the 'perceptual' field is in a state of imbalance or tension. A solution appears in conscious once the perceptual field recognizes itself into a better, more harmonious or balanced state [12]. In a word, these theories all suggest impasses may occur at an early stage.

Existing studies using various approaches [7,12,15,16] have revealed the forming of mental impasses would result in more (attentional) fixation. When problem solvers enter an impasse, they do not know what to do next. So they tend to stare at the problem without testing particular solution ideas [12]. ERP studies revealed a late positive potential (LPP), especially the P3 could better reflect that process [17]. Traditionally, problem-solving, especially insight problem-solving has been viewed as an area of "higher cognition" that is presumed to depend on conscious reasoning and processing. However, work in the last two decades had shown that it could be significantly influenced by nonconscious processes as well, even could be solved without awareness [18]. Moreover, many studies have revealed perceptual features (e.g., problem familiarity, see $[13,19]$ ) which generally triggered unconscious processes also played important roles in insight problem-solving, especially in determining what time mental impasses appear. In daily life, individuals often assess rapidly (usually unconsciously or intuitively) potential probability of a problem which successfully solved through its perceptual features as reflected by P2 (a positive ERP component peaking around $200 \mathrm{~ms}$ after the onset of stimuli). This idea has been strengthened by some recent brain potential studies. In an ERP study, Paynter et al. observed subjects could estimate if the answer was known much faster than the answer could be retrieved and that process was linked with an early fontal P2 epoched from 180 to $280 \mathrm{~ms}$ post-stimuli [18,20]. Ryals et al. further found that the early ERP effect starting at 125 ms was associated with word identification success versus failure. Also, they argued that this early ERP effect could be a preconscious marker of downstream word identification success vs. failure [21]. In that case, the mental impasse may, at least partially, occur at an early stage.

As far, only a prior study performed by our research group has provided some empirical information for the aforementioned argument. The researchers adopted normal three-word Chinese riddles and employed ERPs to capture brain markers of mental impasses. Their results showed that cognitive process underlying the active solutions-seeking of riddles with impasses compared to those without impasses elicited a more positive potential in the time windows of 120-210 ms (P170) and 620-800 ms (late LPP) after the onset of problems. The study suggests that the early P170 is linked with cognitive processes that people perceive intuitively mental impasses at the perceptual stage and the late LPP is associated with a conscious reappraisal and reflec- tion of the impasse [22]. That study has already investigated initially the time course of mental impasses, but there are still many ambiguities. First, although it exhibited the intuition even preconscious awareness play a critical role in insight problem-solving, the characteristics of cognitive processes reflected by the observed electrophysiological effect are unclear. In particular, it is unclear that whether the potential cognitive process represented by the early ERP effect is metcognition or not. Second, previous studies did not provide empirical evidence or data for illustrating the exact relationship between mental impasse and problem difficulty. Finally, cognitive processes reflected by late LPP need empirical analysis and further investigations. Studies on emotion regulation have revealed that cognitive reappraisal usually elicits a LPP [23,24]. In this context, cognitive processes reflected by the late LPP is not clear (i.e., is it a lagged P3 or a LPP linked with cognitive reappraisal?). Based on the aforementioned, the current study applied a source analysis measure to reanalyze and provided some new empirical information for identifying time course of mental impasses underlying insight-like problem solving.

\section{Materials and methods}

\subsection{Subjects}

Thirteen healthy university students with normal or corrected-to-normal visual acuity were tested ( 8 females; aged 23-28 years; right-handed). All gave informed consent to participate.

\subsection{Stimuli}

Similar to previous studies $[11,25]$, visual stimuli consisted of 130 three-character Chinese riddle problems and singlecharacter solutions developed in two recent studies [22,26]. They were selected by three specialists on dimensions of familiarity, visual complexity, phoneme and scored by another 103 subjects (21 male, aged $23.34 \pm 1.94$ years) on a five-point scale in difficulty ("1" very easy, "5" very difficult). About half (e.g., 黄梅天-零; in English, “黄梅天” is "the rainy season", “零” is "zero"; the Chinese word “零” is composed of “雨” (rain) and “令” (season), thus the answer to “黄梅天” is “零”) were somewhat difficult (the mean score ranges from 2.2 to 3.4) and the other half (e.g., 十五 日-胖; in English, “十五日” is "half a month”, “胖” is overweight; the Chinese word “胖” is composed of “月” (moon) and “半” (half), thus the answer to “十五日” is “胖” ) were fairly easy (the mean score ranges from 1.4 to 1.7). All words were high frequency.

\subsection{Procedure}

The subjects were seated at a distance of about $70 \mathrm{~cm}$ from 
a screen centered at eye level in an electrically shielded, sound-attenuating room. The experiment included two parts. In the practice, the subjects were trained with 10 riddles (included 2 false-matched fillers). The formal experiment, consisting of 110 true-matched riddles and 10 fillers, was divided into 3 blocks of 40 riddles each. Subjects were allowed to have an enough rest between blocks. The fillers were used for avoiding response set and excluded from analysis. The stimuli were randomly presented.

Each trial was initiated by the presentation of a black fixation cross in the center of a white display screen. The cross remained for $500 \mathrm{~ms}$, followed by a white screen for $300 \mathrm{~ms}$. The riddle problem subsequently appeared on the screen and persisted for $7000 \mathrm{~ms}$. After a $300 \mathrm{~ms}$ the white screen appeared, it was then followed by a solution presented for a maximum of $4000 \mathrm{~ms}$. During the interval, the subjects should make judgments by pressing a key. If they have worked out a correct solution, they should press " 1 "; if they have not worked out a solution before the solution presentation, and have not formed an association between the problem and the solution, they should press " 3 "; if they have generated an incorrect solution or not generated any solution but formed an association between the solution and problem, they should press "2". Accordingly, the problem types were determined. Problems without impasses (POI) were those problems for which the subjects pressed " 1 "; problems with impasses (PWI) were those problems for which the subjects pressed " 3 ". The problems for which the subjects pressed "2" would be excluded from analysis because it was not clear whether there were impasses or not.

\subsection{ERP recording and analysis}

EEG was continuously recorded via 64 scalp electrodes mounted on an elastic cap (Neuroscan) according to the extended 10-20 system. All recordings were referenced to the right mastoid. Eye blinks were recorded through left supraorbital and infraorbital electrodes. The horizontal electroculogram (EOG) was recorded via electrodes placed $1.5 \mathrm{~cm}$ lateral to the left and right external canthi. All interelectrode impedance was kept less than $5 \mathrm{k} \Omega$. The EEG was amplified using a $0.1-30 \mathrm{~Hz}$ band pass and was continuously sampled at $500 \mathrm{~Hz} / \mathrm{channel}$ for off-line analysis. The EEG data were re-referenced offline to the algebraic average of the left and right mastoids. Ocular artifacts were corrected with an eye-movement correction algorithm. All trials in which EEG voltages exceeded $\pm 90 \mu \mathrm{V}$ during the recording epoch were excluded from the analysis.

Analysis of the ERP data was performed for $800 \mathrm{~ms}$ starting at the onset of the problems. Epochs for every subject were averaged relative to a pre-stimulus baseline that was made up of $100 \mathrm{~ms}$ of activity proceeding the epoch of interest. Trials with artifacts (voltage exceeded $\pm 90 \mu \mathrm{V}$ in any channel) and false-matched problems were excluded from the average. The trials included in any individual av- erage were more than 20. A filter was set to $30 \mathrm{~Hz}$ for low pass after the average. F3, FZ, F4, C3, CZ, C4, P3, PZ, P4, $\mathrm{O} 1, \mathrm{OZ}$, and $\mathrm{O} 2$ were selected for statistical analyses. The scalp sites were divided into two orthogonal factors of caudality (frontal, F; central, C; parietal, P; occipital, O) and laterality (left, L; midline, M; right, R). The average amplitude was computed by a three-way repeated measures analysis of variance (ANOVA). The factors were problem type, caudality, and laterality. The Greenhouse-Geisser correction was applied where appropriate.

\subsection{Source analysis}

A single equivalent dipole source model was used for each independent component selected. The DIPFIT function in EEGLAB [27], a non-linear fitting of a single dipole model, was used to explain the scalp potential distribution [28]. The source location was estimated within a four-shell, spherical model of the head. For the head model, the researchers assumed conductivities $\left(\operatorname{mhos} \mathrm{m}^{-1}\right.$ ) of $0.33,0.0042,1.00$, and 0.33 for the scalp, skull, CSF, and brain, respectively. The radii of the spheres were standardized to $85,79,72$, and 71 $\mathrm{mm}$, respectively. Transposition of the dipole location from the spherical head model to the average MRI template was included in the DIPFIT function, by co-registering the Montreal Neurological Institute (MNI) average brain image with the electrode landmark positions. Then, the anatomical location of the fitted source was defined using the Talairach Daemon client with $5 \mathrm{~mm}$ of cube search range (Research Imaging Center, University of Texas) [29].

\section{Results}

Behavioral results showed the mean trials for PWI, POI, and the discarded " 2 " problems were not different $(37 \pm 11.3$, $30 \pm 11.1$, and $30 \pm 10.8$, respectively), $F(2,24)=1.77, P>0.05$. To investigate the exact relationship between mental impasses and problem difficulty, the present study performed a detailed analysis. Those problems were sorted into two categories according to the predefined problem difficulty (assessed by 103 participants) in the prior experiment. One is difficult, the other is relatively easy. Similarly, the present study counted the number of response of two types, i.e., PWI and POI. As before, problem types were determined by individual subjective responses. Results showed that $69.82 \%$ of problems with mental impasses are the predefined difficult problems, whereas only $30.18 \%$ are the easy ones. Meanwhile, only $10.84 \%$ of problems without mental impasses are the predefined difficult problems, about $89.16 \%$ of them are the easy ones. The correlation analysis further revealed that there is a very significant (positive) correlation (about 0.70 ) between the number of individuals' PWI and the number of individuals' predefined difficult problems (i.e., only involved these predefined difficult and 
easy problems responded with " 1 " and " 3 ", for details, see "2.3 Procedure"), $P<0.001$. Also, there is a highly significant (negative) correlation (about -0.70 ) between the number of individuals' POI with the number of the predefined difficult ones, $P<0.001$. However, there is also a significant (positive) correlation (about 0.24 ) between the number of the PWI and that of the easy, $P<0.05$. Additionally, there is a strongly (positive) correlation $(0.74)$ between the number of the POI and that of the easy, $P<0.001$. These detailed results suggest that problem difficulty also plays an important role in mental impasses, it is not the sole factor determines whether a problem includes a mental impasse or not. The grand average ERPs and topographic maps for each condition are shown in Figure 1. Visual inspection revealed that early ERP components following problems, were markedly elicited, including N1 (N60-120), P2 (P120-210), N2 (N190-250), and N3 (N320-440), independently of problem type. Latencies were not different $\left(P_{\mathrm{s}}>0.05\right)$. The average amplitude of $\mathrm{N} 1$ was measured by the three-way repeated measures ANOVA, only the effect of laterality $\times$ caudality was marked, $F(6,72)=4.08, P<0.05$. Similarly, the ANOVAs revealed no main effect on N2 except significant problem typexcaudality and laterality $\times$ caudality interactions, as well as no difference on N3 except the effect of caudality, $F(3,36)=3.50, P<0.05$, and laterality $\times$ caudality, $F(6,72)=5.50, P<0.01$.

However, distinct ERP waveforms emerged for POI vs. PWI on the P2 (120-210 ms) and within LPP (440-800 ms) time range. As shown in Figure 1, P2 was a positivity occurring approximately at $160 \mathrm{~ms}$ post-stimuli and peaking at $\mathrm{Cz}$. The study employed 2 (problem type) $\times 4$ (caudality) $\times$ 3(laterality) repeated measures ANOVA to the amplitudes of P2 and LPP, respectively. Results on P2 showed significant effects of problem type, $F(1,12)=5.94, P<0.05$, caudality, $F(3,36)=11.75, P<0.001$, and laterality, $F(6$, $72)=14.01, P<0.001$. Further test showed the frontocentral P2 exhibited significant effects of problem type, $F(1,12)=$ $6.49, P<0.05$, laterality, $F(2,24)=7.98, P<0.01$; whereas the parietoccipital $\mathrm{P} 2$ exhibited effects of caudality, $F(1$, $22)=16.50, P<0.01$, laterality, $F(2,24)=8.56, P<0.01$, and laterality $\times$ caudality, $F(2,24)=5.08, P<0.05$. PWI elicited a greater anterior P2 but not posterior P2 than POI. Previous studies revealed there was a difference in some late time frames, the time course of LPP was thus divided into two consecutive time windows: early LPP (440-620 ms) and late LPP (620-800 ms). The analyses showed the frontocentral sites elicited a greater early LPP than the parietoccipital ones, and the late LPP showed significant effects of problem type, $F(1,12)=10.33, P<0.01$, and caudality, $F(3$, 36) $=20.05, P<0.001$. To exclude alternative interpretations on cognitive function of the late LPP (e.g., cognitive reappraisal of mental impasse, because the observed LPP has been reported to be associated with cognitive reappraisal in emotion regulation literature), the present study employed source analysis to capture the neural generators of the late LPP (620-800 ms). The current investigation obtained the difference wave by subtracting the ERP waveform triggered by POI from the ERP waveform elicited by PWI. Independent component analysis (ICA) revealed that the difference wave in the time course of $620-800 \mathrm{~ms}$ has seven neural generators. However, only two cortical generators were relatively reasonable. One was located approximately in the left superior frontal gyrus (location according to Talairach coordinates: $x=-5, y=24, z=50$; Figure 2) and the

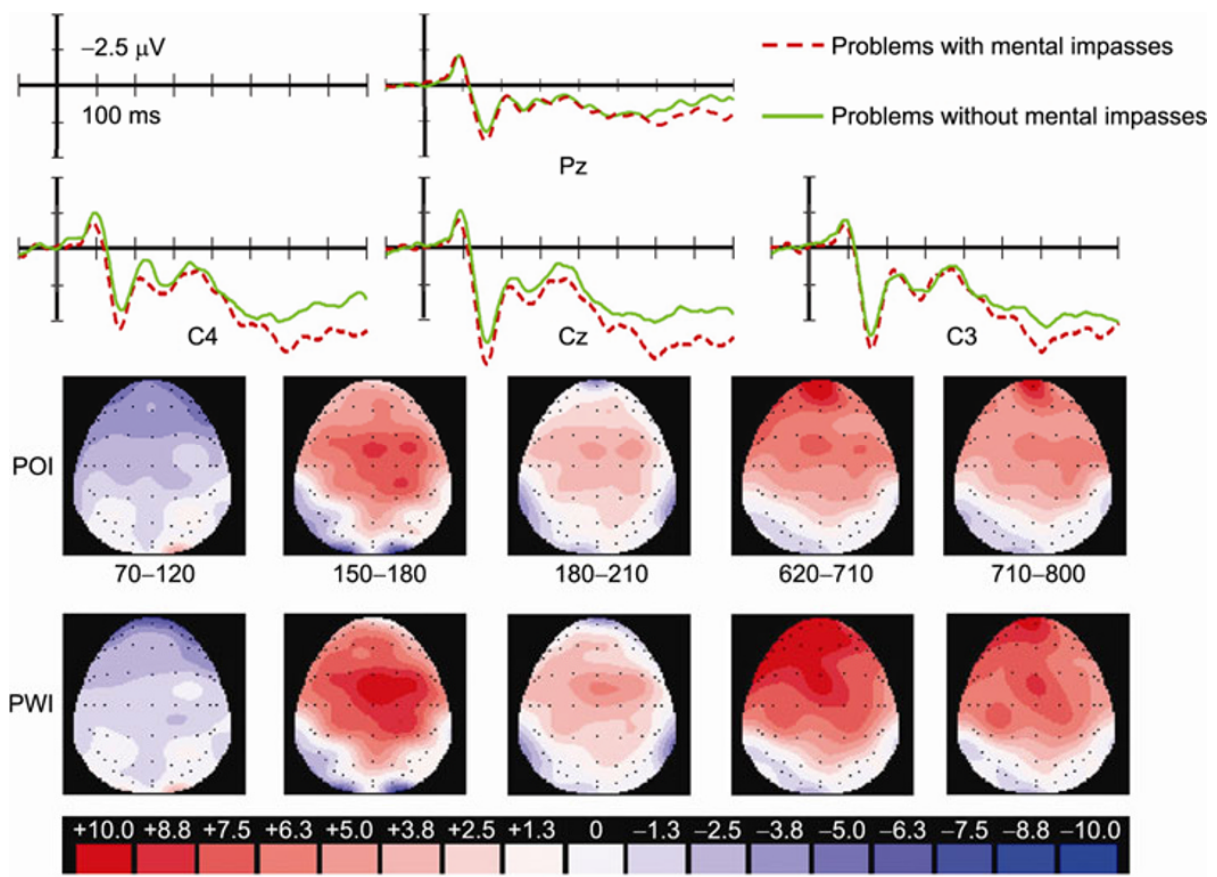

Figure 1 Grand average ERPs $(N=13)$ (top) and topographic maps (bottom, up: POI; down: PWI) on experimental conditions. POI, problems without mental impasses; PWI, problems with mental impasses. 


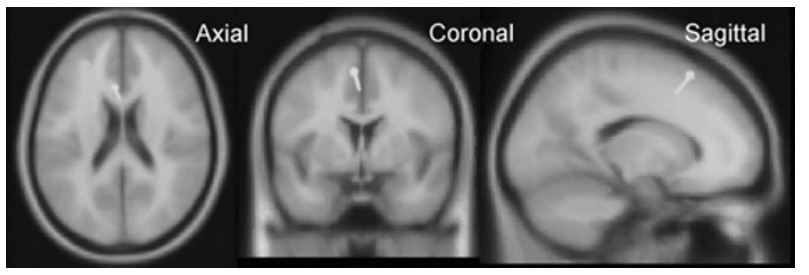

Figure 2 Dipole source localization (left superior frontal gyrus) for the difference waveform (PWI-minus-POI) of the late LPP (620-800 ms) was superimposed on the MR-based head model of one subject.

second near the left cuneus $(x=0, y=-94, z=24)$. However, the first was relatively better than the second, because the residual variance (RV) of the first came close to $0.12 \%$ whereas the RV of the second was $32.51 \%$.

\section{Discussion}

The mental impasse plays a necessary and critical role in a variety of insight problem solving and incubation, but, as yet, relatively little is known about its neural correlates. Moreover, the question when the impasse occurs during insight problem solving has rarely been studied. The study adopted ERPs to systematically investigate temporal dynamics of an impasse for the first time.

The most important finding of the study is that the frontocentral P2 emerged earlier in peak latency (than that of the classical P2 observed in previous studies), and was larger in amplitude for problems with impasses (PWI) than for problems without impasses (POI). The findings reveal the P2 peaking at approximately $160 \mathrm{~ms}$ post-stimulus as a function of problem type; PWI elicited a larger P2 than POI. P2 has generally been associated with perceptual processing of stimuli and priming effect. Here, the observed P2 amplitude solely exhibited a problem type effect in the frontocentral areas but not in the parietoccipital areas, indicating the $\mathrm{P} 2$ reflected not only neuropsychological processing of perception. Although the problems in this study were divided into difficult and easy problems and one might believe the frontocentral P2 amplitude reflected neurocognitive processing of problem difficulty, previous studies using various types of problems revealed problem difficulty was marked by the amplitude of N2 or N300 [30,31], even the latencies of ERP components [31-33]. With regard to the relationship between mental impasses and problem difficulty, the present study made detailed analyses. Results showed that problem difficulty also played an important role in mental impasses, it was not the sole factor determined whether a problem included a mental impasse or not. In other word, the current results implied that not only problem difficulty but also other factors such as individual's knowledge and problem representation played important roles in determining whether an individual might encounter a mental impasse or not. In this context, the $\mathrm{P} 2$ did not reflect cognitive process of difficulty. Therefore, the P2 may reflect neurocognitive pro- cess of mental impasses.

It was worth thinking that the frontocentral $\mathrm{P} 2$ reflected the final formation of mental impasses, although some studies argued individuals have already initiated the generation of impasses before they see or perceive problems [22]. The impasses of some types of problems occur without any exploration of problem space, but it occurs after an initial exploration of problem space for most types of problems [12]. It is impossible for the initial exploration of the problem space (which has to occur before an impasse) to take place in such an immediate period following problem onset. Accordingly, the observed $\mathrm{P} 2$ probably reflected metacognitive processing of impasses but not the final formation of the impasse itself. Traditionally, metacognition aids in the representation of the problem through the conscious reflection and reassessment of the problem when one encounters a piece of mental obstacle. Given it is impossible for the P2 to represent the reflective or conscious thinking on the forming or formed impasses; the P2 thus reflects metacognitive awareness of an impasse. In other words, metacognition in this study may not be responsible for off-line reflection and conscious reassessment but initial evaluation as well as rapid assessment without conscious. Specifically, the P2 may play a role by performing rapid assessment of whether a stimulus seems sufficiently familiar to merit a successful or effortful solving. Problems that pass this initial assessment may encounter potential impasses while those deemed too unfamiliar may not. Put another way, the neurocognitive process reflected by frontocentral P2 uses a rapid heuristic based on the stimulus-driven processing to assess (or differentiate) initially potential impasses so that guides strategy selection and attention allocation in the present task. In according with this view, Paynter et al. found an early frontal P2 effect (beginning at $180 \mathrm{~ms}$ ) was associated with an initial FOK judgment that discriminated between studied math equations that would later be solved and studied math equations that would not, and the P2 amplitude as a function of problem familiarity (collapsed across strategy chosen) and whether or not the subject obtained a successful on-time retrieval trial [19]. The frontal P2 as a preconscious indicator of FOK was thought to be linked with the use of a rapid heuristic based on the perceptual processing of a stimulus to guide strategy selection. Ryals et al. observed an early ERP effect starting at $125 \mathrm{~ms}$ associated with word identification success versus failure [21]. Nikolaev et al. also found that a frontal P2 evoked by the effect-incompatible flankers might reflect stimulus evaluation and conflict detection, and thought that the P2 effect might be involved in performing a quick assessment of whether a stimulus seemed sufficiently familiar to merit an effortful search [34]. Previous neuroimaging studies revealed there was an "early warning system" involving mental impasses during insight problem solving $[35,36]$. Furthermore, the "early warning system" exhibited different activations when the subjects became familiar with the insightful solving of 
puzzles and developed some task-general strategies [35]. In another ERP study [22], the researchers found that intuition played an important role in foreseeing the impasse. Taken together, the findings demonstrate the $\mathrm{P} 2$ in processing insight problems, as a stimulus-driven frontal metacognitive mechanism, reflects preconscious awareness of the impasse. It suggests metacognition and conventional thinking of human may have so high a sensitivity and capacity of automatic information processing that individuals can know whether there is a potential impasse underlying problem solving before they start thinking about problems.

Another finding of interest was that a broadly distributed positivity between 620 and $800 \mathrm{~ms}$ was elicited by two types of problems, and the amplitude differences across conditions were mainly pronounced at central and frontal sites, although there was no problem type effect in the time window of early LPP (from 440 to $620 \mathrm{~ms}$ ). Our experiment revealed the LPP might not be linked with set shifts or the forming of novel associations mentioned in other studies on insight, because the data suggested subjects had never broken mental sets or formed novel associations until the solution presentation in the PWI condition. Chen et al. found LPP might consist of several separate components, including the anterior $\mathrm{P} 3 \mathrm{a}$ and posterior $\mathrm{P} 3 \mathrm{~b}$ and the positive slow wave under certain conditions [32]. According to the results of source analysis, the neural generator of the late LPP was located in the left superior frontal gyrus. Previous studies have exhibited that the neural generator of P3a was primarily located in the frontal cortex $[37,38]$ whereas the P3b was mainly located near the superior parietal lobule [38,39]. Thus, the observed LPP (in the time window of 620-800 ms) is actually a P3a component. It has generally been accepted that $\mathrm{P} 3 \mathrm{a}$ is a late phase of orienting response that is sensitive to central controlled processes [32,40]. In particular, P3a is associated with the controlled processing phenomena triggered by previous automatic processes reflected by the frontocentral P2, and its generation requires top-down mechanism initiated by frontal lobe functions [40]. That is, attention marked by the $\mathrm{P} 3 \mathrm{a}$ is selective and conscious. Here, the participants (selectively) focus their attentions on riddle problems rather than others.

The problem solver is likely to experience an impasse resulting from mental set and/or exhausting the available options and resources, since he/she is unable to make further progress and subsequently he/she will give up. From an information processing point of view, the system has reached a limited point at mental impasse: any new possible options or interpretations from long-term memory are blocked from further processing within working memory. As mentioned in the introduction, attention usually serve as a "general gatekeeper" for manipulating information within working memory by exerting top-down control over which information is the most relevant and may occupy the limited space [41]. In other words, the impasse may be caused by an "attentional overload" [16]. Indeed, numerous studies observed that mental impasses were linked with attention.
Thomas and Lleras observed post-impasse attentional shifts function as an implicit aid to insight [42]. Knoblich et al. observed the problem solver spent more time to stare at the problem after they entered into an impasse [12], i.e., mental impasses have been linked to prolonged periods of visual fixation. Bilalić also found problem solvers continued to stare at features of the problem related to the solution they had already thought of [43]. In an ERP study using verbal remote associate problems, the investigators found selective attention was linked with impasses [16]. Additionally, previous studies have illustrated that P3 is a good ERP marker of selective attention [17]. Therefore, the late LPP may reflect the forming of an impasse.

In sum, mental impasse is a necessary component for insight $[6,12]$ and that the time period of struggling with the problem without achieving the solution is as important as the sudden realization [16]. To the best of our knowledge, no study has assessed the time course of mental impasses. Using real-time ERPs, the study investigated temporal dynamics of impasses underlying insight-like problem solving. Our findings that the impasse is associated with the frontocentral P2 and the late LPP post problems demonstrate the impasse occurs at a relatively early stage. Furthermore, the study revealed that metacognition as an "early warning system" based on perceptual features involves guiding strategy selection and attention allocation. Additionally, it is noteworthy that frontal regions and perceptual features of problems play an important role in differentiating potential impasses during insight problem solving. In a word, the present study demonstrates the impasse appears initially at a relatively early stage of problem solving, although it can not rule out the possibility that mental impasses underlying some types of insight problem solving happen at a traditionally later stage. Studies in future seem to further investigate the accuracy of the initial or experience-based metacognitive prediction on the impasse and the role of intuition in insight-like problem solving.

We thank Prof. Knoblich G. and Liu Chao, whose comments and suggestions prompted further analysis and formed this research. This work was supported by the National Basic Research Program of China (2010CB833904), Research Innovation Program for College Graduates of Jiangsu Province (CXLX12_0353, CXLX12_0351), and the Fourth High-level Personnel Training Project in Jiangsu Province.

1 Dietrich A, Kanso R. A review of EEG, ERP, and neuroimaging studies of creativity and insight. Psychol Bull, 2010, 136: 822-848

2 Jung-Beeman M, Bowden E M, Haberman J, et al. Neural activity when people solve verbal problems with insight. PLoS Bio, 2004, 2: e97

3 Ludmer R, Dudai Y, Rubin N. Uncovering camouflage: amygdala activation predicts long-term memory of induced perceptual insight. Neuron, 2011, 69: 1002-1014

4 Luo J, Niki K. Function of hippocampus in "insight" of problem solving. Hippocampus, 2003, 13: 316-323

5 Kaplan C A, Simon H A. In search of insight. Cogn Psychol, 1990, 22: $374-419$

6 Moss J, Kotovsky K, Cagan J. The effect of incidental hints when problems are suspended before, during, or after an impasse. J Exp Psychol Learn Mem Cogn, 2011, 37: 140-148 
7 Smith S M. Getting into and out of mental ruts: A theory of fixation, incubation, and insight. In: Sternberg R J, Davidson J E, eds. The Nature of Insight. Cambridge MA: MIT Press, 1995. 229-251

8 Weisberg R W, Alba J W. An examination of the alleged role of "fixation" in the solution of several "insight" problems. J Exp Psychol Gen, 1986, 110: 169-192

9 Zhao Y, Tu S, Lei M, et al. The neural basis of breaking mental set: an event-related potential study. Exp Brain Res, 2011, 208: 181-187

10 Qiu J, Li H, Luo Y, et al. Brain mechanism of cognitive conflict in a guessing Chinese logogriph task. Neuroreport, 2006, 17: 679-682

11 Qiu J, Li H, Yang D, et al. The neural basis of insight problem solving: An event-related potential study. Brain Cogn, 2008, 68: 100-106

12 Knoblich G, Ohlsson S, Raney G. An eye movement study of insight problem solving. Mem Cogn, 2001, 29: 1000-1009

13 Knoblich G, Ohlsson S, Haider H, et al. Constraint relaxation and chunk decomposition in insight problem solving. J Exp Psychol Learn Mem Cogn, 1999, 25: 1534-1555

14 Ollinger M, Jones G, Knoblich G. Investigating the effect of mental set on insight problem solving. Exp Psychol, 2008, 55: 269-282

15 Chrysikou E G, Weisberg R W. Following the wrong footsteps: Fixation effects of pictorial examples in a design problem-solving task. J Exp Psychol Learn Mem Cogn, 2005, 31: 1134-1148

16 Sandkühler S, Bhattacharya J. Deconstructing insight: EEG correlates of insightful problem solving. PLoS ONE, 2008, 3: e1459

17 Rushby J A, Barry R J, Doherty R J. Separation of the components of the late positive complex in an ERP dishabituation paradigm. Clin Neurophysiol, 2005, 116: 2363-2380

18 Paynter C A, Kieffaber P D, Reder L M. Problem-solving without awareness: An ERP investigation. Neuropsychologia, 2010, 48: 3137- 3144

19 Reder L M, Ritter F. What determines initial feeling of knowing? Familiarity with question terms, not with the answer. J Exp Psychol Learn Mem Cogn, 1992, 18: 435-451

20 Paynter C A, Reder L M, Kotovsky K. Knowing we know before we know: ERP correlates of initial feeling-of-knowing. Neuropsychologia, 2009, 47: 796-803

21 Ryals A J, Yadon C A, Nomi J S, et al. When word identification fails: ERP correlates of recognition without identification and of word identification failure. Neuropsychologia, 2011, 49: 3224-3237

22 Shen W B, Liu C, Luo J, et al. Brain perceived intuitively mental impasses in insight problem solving: An ERP study (in Chinese). Acta Psychol Sin, 2012, 44: 924-935

23 Thiruchselvam R, Blechert J, Sheppes G, et al. The temporal dynamics of emotion regulation: An EEG study of distraction and reappraisal. Biol Psychol, 2011, 87: 84-92

24 Hajcak G, Nieuwenhuis S. Reappraisal modulates the electrocortical response to unpleasant pictures. Cogn Affective Behav Neurosci, 2006, 6: 291-297

25 Mai X Q, Luo J, Wu J H, et al. “Aha!” effects in a guessing riddle task: An event-related potential study. Hum Brain Mapp, 2004, 23: $128-128$
26 Shen W B, Liu C, Zhang X J, et al. The time course and hemispheric effect of "insight" in three-character Chinese riddles task: An ERP study (in Chinese). Acta Psychol Sin, 2011, 43: 229-240

27 Delorme A, Makeig S. EEGLAB: An open source toolbox for analysis of single-trial EEG dynamics. J Neurosci Meth, 2004, 134: 9-21

28 Scherg, M. Fundamentals of dipole source potential analysis. In: Grandori F, Hoke M, Romani G, eds. Auditory Evoked Magnetic Fields and Electric Potentials. Karger: Basel, 1990. 40-69

29 Lancaster J L, Woldorff M G, Parsons L M, et al. Automated Talairach atlas labels for functional brain mapping. Hum Brain Mapp, 2000, 10: 120-131

30 Zhou X, Chen C, Dong Q, et al. Event-related potentials of singledigit addition, subtraction, and multiplication. Neuropsychologia, 2006, 44: 2500-2507

31 Berg W K, Byrd D L, McNamara J P, et al. Deconstructing the tower: Parameters and predictors of problem difficulty on the Tower of London task. Brain Cogn, 2010, 72: 472-482

32 Chen A, Luo Y, Wang Q, et al. Electrophysiological correlates of category induction: PSW amplitude as an index of identifying shared attributes. Biol Psychol, 2007, 76: 230-238

33 Pauli P, Lutzenberger W, Birbaumer N, et al. Neurophysiological correlates of mental arithmetic. Psychophysiology, 1996, 33: 522529

34 Nikolaev A R, Ziessler M, Dimova K, et al. Anticipated action consequences as a nexus between action and perception: Evidence from event-related potentials. Biol Psychol, 2008, 78: 53-65

35 Luo J, Niki K, Phillips S. The function of the anterior cingulate cortex (ACC) in the insightful solving of puzzles: the ACC is activated less when the structure of the puzzle is known. J Psychol Chin Soc, 2004, 5: 195-213

36 Luo J, Niki K, Phillips S. Neural correlates of the 'Aha! reaction'. Neuroreport, 2004, 15: 2013-2017

37 Friedman D, Cycowicz Y M, Gaeta H. The novelty P3: An eventrelated potential (ERP) sign of the brain's evaluation of novelty. Neurosci Biobehav Rev, 2001, 25: 355-373

38 Restuccia D, Marca G D, Marra C, et al. Attentional load of the primary task influences the frontal but not the temporal generators of mismatch negativity. Brain Res, 2005, 25: 891-899

39 Halgren E, Marinkovic K, Chauvel P. Generators of the late cognitive potentials in auditory and visual oddball tasks. Electroenceph clin Neurophysiol, 1998, 106: 156-164

40 Carretié L, Hinojosa J A, Martín-Loeches M, et al. Automatic attention to emotional stimuli: Neural correlates. Hum Brain Mapp, 2004, 22: 290-299

41 Awh E, Vogel E K, Oh S H. Interactions between attention and working memory. Neuroscience, 2006, 139: 201-208

42 Thomas L, Lleras A. Covert shifts of attention function as an implicit aid to insight. Cognition, 2009, 111: 168-174

43 Bilalić M, McLeod P, Gobet F. Why good thoughts block better ones: the mechanism of the pernicious Einstellung (set) effect. Cognition, 208, 108: 652-661

Open Access This article is distributed under the terms of the Creative Commons Attribution License which permits any use, distribution, and reproduction in any medium, provided the original author(s) and source are credited. 